1 Title: Adolescence as a sensitive period of brain development

2

3 Authorship: Fuhrmann, D. ${ }^{1 *}$, Knoll, L. J. ${ }^{1}$, \& Blakemore S. J. ${ }^{1}$

$4{ }^{1}$ Institute of Cognitive Neuroscience, Division of Psychology and Language Science,

5 University College London, WCIN 3AR, London, UK

6 * Please address correspondence concerning this article to Delia Fuhrmann, Institute of

7 Cognitive Neuroscience, Division of Psychology and Language Science, University College

8 London, WCIN 3AR, London, UK. Email: delia.fuhrmann.13@ucl.ac.uk

9

10 Keywords: sensitive period, adolescence, neuroplasticity, mental health, stress, training 
11 Abstract: The human brain undergoes substantial changes in adolescence, especially in

frontal, parietal and temporal cortices. It has been proposed that these changes in brain structure and function are characterised by relatively high levels of plasticity, making adolescence a sensitive period of development for environmental influences such as drugs, stress or cognitive training. Drugs, such as cannabis, have a particularly deleterious effect on cognitive performance and brain function during adolescence, and social stress during this period of life confers long-lasting negative effects on mental health. Heightened plasticity in adolescence might lead not only to increased vulnerabilities. Plasticity in cognitive control and memory performance during this period of life might also be heightened, making adolescence a window of opportunity for education.

\section{Brain development in adolescence}

Neuroimaging studies in the past two decades have demonstrated that the human brain undergoes protracted development, including during adolescence, the period of life that starts at puberty and ends at the point at which an individual attains an independent role in society $[1,2]$.

White matter volume and integrity increases throughout childhood and adolescence into adulthood. The pattern of increase differs between brain regions with frontal and temporal regions showing particularly pronounced changes in adolescence [3]. White matter volume increases are thought to reflect an increase in axonal calibre [4] or myelination [5, 6]. Myelin acts as an electrical insulator of high resistance and low capacitance, which increases signal conduction velocity [7]. 
Grey matter consists mainly of neuronal cell bodies, glia, dendrites and synapses. In many cortical regions, grey matter volume increases from infancy through childhood, then declines throughout adolescence and into the twenties [6]. Grey matter volume undergoes particularly substantial decreases in frontal and temporal grey matter during adolescence [8]. It has been proposed that the reduction in grey matter during adolescence is due to a number of factors, including increasing white matter encroaching on grey matter [3], environmentally-driven synaptic pruning [9], and a reduction in glia [10].

The ongoing development in white and grey matter during adolescence is accompanied by marked changes in cognition. Piaget conceptualized adolescence as a formal operational stage of development during which individuals increasingly rely on abstract thought and reasoning [11]. This dovetails with recent evidence from Diffusion Tension Imaging (DTI) studies suggesting that adolescent white matter maturation in frontal and parietal regions and their connections is associated with improvements in IQ [12] and working memory performance [13]. Similarly, grey matter reductions in frontal and parietal regions as well as working memory during adolescence [14] and thinner parietal cortices in early adolescence predict better problem solving, planning and verbal learning [15]. Social cognition also undergoes pronounced changes during this period of life, including significant maturation of perspective taking [16] and face processing [17] during human adolescence.

52 The evidence for the reorganisation of brain structure and cognition during adolescence has led to the suggestion that adolescence is a sensitive period of brain development $[18,19]$. It has been proposed that neural plasticity, the way the brain adapts to internal or external changes, is heightened, rendering the adolescent brain particularly susceptible to 
environmental input. We will explore three areas in which adolescence is particularly likely to be characterised by heightened plasticity: the effects of drug use; the social environment; and cognitive control and memory.

\section{The effects of drug use on adolescent brain development}

The developing brain may be particularly sensitive to drugs such as cannabis. Cannabis is one of the most widely recreationally used drugs among adolescents and adults in the US and UK $[20,21]$. Cannabinoid exposure during early adolescence is thought to initiate neuroplasticity, resulting in lasting changes in brain structure and cognitive deficits [22, 23]. A recent study suggested that significant grey matter atrophy in the adult temporal pole, parahippocampal gyrus and insula was linked to heavy cannabis consumption during adulthood or adolescence, or moderate (recreational) use before the age of 18 [24]. Longitudinal data indicated that self-reported persistent cannabis use between 13 and 15 years of age was associated with a significant decline in cognitive abilities [25]. See Figure 1. The longer the period of cannabis consumption, the greater the decline in cognitive abilities [25]. This cognitive decline was more pronounced for participants who used cannabis before age 18 as compared to after. It should be noted that alternative explanations, such as preexisting mood or anxiety disorders mediating both cannabis-use and cognitive problems, cannot be ruled out [26].

Molecular and cellular data on the effects of cannabis in adolescence is scarce and it is not yet clear what makes the developing brain particularly sensitive to cannabis. Cannabis affects the endocannabinoid system, which, along with other neurotransmitter systems (e.g. the glutamatergic and dopaminergic systems), undergoes extensive restructuring during 
adolescence [27]. Cannabis may disturb neurodevelopmental processes known to be mediated by the endocannaboid system, including neuronal genesis, neural specification, neuronal migration, axonal elongation and glia formation [28-30].

82 Cannabis use during adolescence may increase the risk of developing psychotic disorders such as schizophrenia [31-33]. Research has shown that individuals with a genetic predisposition to schizophrenia and a history of cannabis use are at higher risk of developing schizophrenia compared to those without [34]. Animal models have suggested a causal link between first-time cannabis consumption in adolescence and schizophrenic-like symptoms. Cannabinoid exposure in adolescent rodents predicted schizophrenia-like symptoms such as long-term cognitive deficits in adulthood (e.g. object recognition memory), whereas similar exposure in adult rodents was not linked to such symptoms [35-38].

Recent studies in humans and animals support the notion as adolescence as a period of particular sensitivity to cannabis consumption compared to adulthood. More studies investigating the effect of cannabis during development are needed, however. Sensitivity to cannabis during childhood remains unclear. As cannabis is one of the most widely recreationally used drugs and consumption is typically initiated during adolescence, it is important to understand the impact of cannabis use for social and cognitive development during this time. 


\section{Adolescence as a sensitive period for social stress}

Adolescents are especially sensitive to the social environment, particularly to the influence of peers. Peers influence risk taking behaviours such as drug use and academic performance [40]. Social stress and social exclusion have a significant impact on adolescents [41], and peer victimization and lack of social support has particularly detrimental effects for mental health [42].

Studying rodents provides the opportunity to manipulate experimentally exposure to social stress, and has provided valuable insights into the deleterious effects of stress in adolescence. Adolescent rats respond differently to social stress compared to adult and juvenile rats $[43,44]$. Adolescent rats subjected to repeated defeat by a dominant individual present with different behavioural patterns (more avoidance rather than aggression), and recover less from renewed stress, compared with adult rats. Exposure to stress in adolescence in rats (compared with adulthood) was also associated with less neuronal activation in areas of the prefrontal cortex, cingulate and thalamus [44]. Social deprivation in rats has been shown to have irreversible effects on some aspects of exploratory behaviour, but only if the deprivation occurs between late childhood and midadolescence (postnatal day 25-45), but not after 45 days [43]. This early study is also one of the few to investigate plasticity in the juvenile, adolescent and adult period (see textbox 'Models of plasticity in adolescence'). 


\section{Models of plasticity in adolescence}

Unless pre-pubertal as well as adult groups are compared to adolescents, the question of whether adolescence is a sensitive period cannot be assessed. There are several possible plasticity profiles $[45,46]$. Adolescence may be stand-alone period of heightened plasticity in certain domains, before and after which plasticity is lower (Model A, Figure 2). Alternatively, childhood and adolescence might form a continuous sensitive period after which plasticity declines (Model B, Figure 2). A third possibility is that plasticity may decline more or less continuously from childhood through adolescence and into adulthood (Model C, Figure 2). In this case adolescence would not be categorised as a sensitive period even though plasticity is heightened as compared with adulthood.

\section{Stress and mental health in adolescence}

Many mental illnesses have their onset in adolescence and early adulthood [47, 48]. See

Figure 3. A representative, longitudinal study showed that $73.9 \%$ of adult cases with a mental disorder have already had a diagnosis before 18 years of age and $50.0 \%$ before 15 years of age [49]. It is thought that psychiatric disorders may in part be triggered by stressexposure in childhood or adolescence [19]. The experience of acculturation stress by immigrant-origin adolescents in US-American schools, for instance, has been shown to predict longitudinally internalizing symptoms such as depression and anxiety [50].

For psychiatric disorders such as post-traumatic stress disorder (PTSD), stress may persist even if the stressor is no longer present. Fear extinction learning is key for a healthy response to stress and the basis for desensitization treatments for PTSD [51]. Fear extinction learning has been found to be attenuated in adolescence as compared to childhood and adulthood - both in humans and in mice [51]. The rodent data in the study indicated that a lack of synaptic plasticity in the ventro-medial prefrontal cortex during adolescence is associated with decreased fear extinction. 
Adolescence is not a clear-cut period of vulnerability to stress, however. In some cases, adolescent animals may show higher resilience to certain stressors than adults [52] and social stress in adolescence can be buffered if rats are socially housed after exposure to stress [53]. Early and targeted mental health interventions aimed at strengthening resilience and providing support during adolescence may help buffer the effects of social stress and bullying, which may in turn improve life-long mental health outcomes.

\section{Adolescence as a sensitive period for cognitive control and memory}

The protracted development in frontal and parietal regions has been linked to changes in cognitive control, including planning [54], measures of executive function and working memory [55]. Mnemonic abilities also generally increase from childhood, through adolescence and into adulthood [56]. Aspects of memory requiring strategic, effortful components are usually found to develop later than those that require less cognitive control [57].

\section{Plasticity in working memory}

Working memory (WM), the ability to hold and manipulate information [58], has been shown to undergo changes beyond childhood. While basic aspects of spatial WM may reach maturity in childhood, complex spatial WM abilities continue to improve during early adolescence [55]. WM tasks recruiting frontal areas show protracted development throughout adolescence [59].

There is some evidence for plasticity of WM in development. For children and young adolescents, gains in WM training, but not knowledge-based training, transferred to 
improvements in fluid intelligence [60]. Improvements were sustained over a 3-month period during which time no further training was implemented. WM training may also be effective in adolescents with poor executive functioning, as well as in typically-developing controls [61]. However, we do not yet know how effects of training differ in adolescents as compared to children or adults, which limits conclusions for adolescence as a sensitive period for WM.

\section{Memory in adolescence and the reminiscence bump}

Memory capacities appear to be heightened in adolescence. The number of autobiographic memories recalled at age 35 or after shows a peak in adolescence, a phenomenon referred to as the reminiscence bump [62]. The lifespan retrieval curve (Figure 4) shows a period of childhood amnesia before around 5 years when autobiographic memories are virtually absent [63]. Memories then increase and reach a maximum between 10 and 30 years, which is followed by a period of fewer recalled memories. Recency effects lead to a better recall of events in the later decades of life. The reminiscence bump is remarkably robust and shows a similar pattern when tested with different mnemonic tests and in different cultures $[62,63]$.

In addition to autobiographical events, the recall of music, books, films and public events from adolescence is also superior compared with from other periods of life [64, 65]. Even mundane events that happened in adolescence and early adulthood appear to be overrepresented in memory, suggesting that mnemonic capacity is heightened during this time of life [66]. A large-scale study showed a peak of other aspects of memory like verbal and visuospatial memory between 14 and 26 years of age [67].

Future studies are required that manipulate experimentally environmental input in child, adolescent and adult groups. Developmental training studies in which different aged 
participants undergo cognitive training and effects are compared to active control groups that receive placebo training may be particularly useful here [68]. Such studies may also 179 directly inform clinical and educational interventions.

\section{Conclusion}

182 Adolescence is a period of protracted brain development that is characterised by gross transformations in white and grey matter that are concomitant with changes in socioemotional and cognitive processing. The development of some of these processes may be particularly susceptible to environmental influences, such as drugs, social stress or cognitive training, making adolescence a sensitive period of development.

The findings discussed here highlight the importance of adolescent health care and education. It has been estimated that $40 \%$ of the world's teenagers do not have access to secondary school education [69]. Even in countries that have compulsory education, schooling often ends between 14 and 16 years of age [70]. In Western countries, such as the UK or US, much attention and resources have been devoted to early development, sometimes creating the impression that experiences in the first few years of life determine lifelong health, education and social outcomes $[71,72]$. This status quo is now changing, however, and heightened awareness is emerging of the importance of later stages in development. A recent WHO report argues for the importance of adolescence for worldwide health [73] and a UK Royal Society report underscored the significance of STEMsubjects education post-16 for the national economy [74]. 


\section{Outstanding questions}

- What are the mechanisms of plasticity in adolescence? How do they interact?

- How do environmental influences impact grey and white matter development in humans?

- Is there greater plasticity in adolescence than in other periods of development or do we see a continuous decline of plasticity from childhood to adulthood?

- Which socio-cognitive processes show particular plasticity in adolescence?

- What are the consequences of stress in humans across the lifespan?

- What are the effects of enrichment and training in adolescence as compared to other age groups?

- Can we harness adolescent plasticity for educational and clinical interventions? Do training effects transfer to real-life measures such as academic performance? If so, what are the side-effects of such interventions? What are the ethical implications of cognitive enhancement through training?

Acknowledgement: We would like to thank Kathryn Mills for comments on an early draft.

\section{References}

1 Damon, W. (2004) In Handbook of Adolescent Psychology (2nd edn) (Lerner, R.M. and

2 Blakemore, S.J. (2012) Imaging brain development: the adolescent brain. Neuroimage

3 Tamnes, C.K. et al. (2010) Brain maturation in adolescence and young adulthood: 
4 Paus, T. (2010) Growth of white matter in the adolescent brain: myelin or axon? Brain Cognition 72, 26-35

5 Gibson, E.M., et al. (2014) Neuronal activity promotes oligodendrogenesis and adaptive myelination in the mammalian brain. Science 344, 1252304

6 Bartzokis, G., et al. (2001) Age-related changes in frontal and temporal lobe volumes in men: a magnetic resonance imaging study. Arch. Gen. Psychiatry 58, 461-465

7 Morell, P. and Quarles, R.H. (1999) The myelin sheath. In Basic Neurochemistry: Molecular, Cellular and Medical Aspects (6 edn) (Siegel, G.J., et al., eds), LippincottRaven

8 Tamnes, C.K., et al. (2013) Brain development and aging: overlapping and unique patterns of change. Neuroimage $68,63-74$

9 Giedd, J.N., et al. (1999) Brain development during childhood and adolescence: a

12 Schmithorst, V.J. et al. (2005) Cognitive functions correlate with white matter architecture in a normal pediatric population: a diffusion tensor MRI study. Hum. Brain Map. 26, 139-147

13 Ostby, Y., et al. (2011) Morphometry and connectivity of the fronto-parietal verbal working memory network in development. Neuropsychologia 49, 3854-3862

14 Tamnes, C.K. et al. (2013) Longitudinal working memory development is related to structural maturation of frontal and parietal cortices. J. Cogn. Neurosci. 25, 1611-1623 
15 Squeglia, L.M., et al. (2013) Early adolescent cortical thinning is related to better neuropsychological performance. J. Int. Neuropsychol. Soc. 19, 962-970

16 Sebastian, C.L., et al. (2012) Neural processing associated with cognitive and affective Theory of Mind in adolescents and adults. Soc. Cogn. Affec. Neurosci. 7, 53-63

17 Cohen Kadosh, K., et al. (2013) Differential face-network adaptation in children, adolescents and adults. Neuroimage $69,11-20$

18 Blakemore, S.J. and Mills, K.L. (2014) Is adolescence a sensitive period for sociocultural processing? Annu. Rev. Psychol. 65, 187-207

19 Andersen, S.L. and Teicher, M.H. (2008) Stress, sensitive periods and maturational events in adolescent depression. Trends Neurosci. 31, 183-191

20 The NHS Information Centre (2011) Statistics on Drug Misuse. NHS

21 Johnston, L.D. et al. (2013) Monitoring the future. National survey results on drug use 1975-2012. Institute for Social Research, University of Michigan

22 Ehrenreich, H., et al. (1999) Specific attentional dysfunction in adults following early start of cannabis use. J. Psychopharmacol. 142, 295-301

23 Pope, H.G. et al. (2003) Early-onset cannabis use and cognitive deficits: what is the nature of the association? Drug and Alcohol Depen. 69, 303-310

24 Battistella, G., et al. (2014) Long-term effects of cannabis on brain structure. J. Neuropsychopharmacol. 39, 2041-2048

25 Meier, M.H. et al. (2012) Persistent cannabis users show neuropsychological decline from childhood to midlife. PNAS 109, E2657-E2664

26 Blakemore, S.J. (2013) Teenage kicks: cannabis and the adolescent brain. Lancet 381, 888-889 
27 Malone, D.T. et al. (2010) Adolescent cannabis use and psychosis: epidemiology and neurodevelopmental models. Br. J. Pharmacology, 2010. 160(3): p. 511-522

28 Berghuis, P., et al. (2007) Hardwiring the brain: endocannabinoids shape neuronal connectivity. Science 316, 1212-1216

29 Harkany, T., et al. (2008) Endocannabinoid functions controlling neuronal specification during brain development. Mol. Cell. Endocrinol. 286, S84-S90

30 Oudin, M.J., et al. (2011) Endocannabinoids regulate the migration of subventricular zone-derived neuroblasts in the postnatal brain. J. Neurosci. 31, 4000-4011

31 D'Souza, D.C., et al. (2009) Cannabis and psychosis/schizophrenia: human studies. Eur. Arch. Psychiatry Clin. Neurosci. 259, 413-431

32 Evins, A.E., et al. (2012) The effect of marijuana use on the risk for schizophrenia. J. Clin. Psychiatry 73, 1463-1468

33 Rubino, T. and Parolaro, D. (2014) ) Cannabis abuse in adolescence and the risk of psychosis: a brief review of the preclinical evidence. Prog. Neuropsychopharmacol. Biol. Psychiatry 52, 41-44

34 Hall, W., et al. (2004) Cannabis use and psychotic disorders: an update. Drug Alcohol Rev. $23,433-443$

35 O'Shea, M. et al. (2004) Chronic cannabinoid exposure produces lasting memory impairment and increased anxiety in adolescent but not adult rats. J. Psychopharmacol. $18,502-508$

36 Quinn, H.R. et al. (2008) Adolescent rats find repeated Delta(9)-THC less aversive than adult rats but display greater residual cognitive deficits and changes in hippocampal protein expression following exposure. J. Neuropsychopharmacol. 33, 1113-1126 
37 Renard, J. et al. (2013) Long-term cognitive impairments induced by chronic cannabinoid exposure during adolescence in rats: a strain comparison. J. Psychopharmacol. 225, 781790

38 Schneider, M. and Koch, M. (2003) Chronic pubertal, but not adult chronic cannabinoid treatment impairs sensorimotor gating, recognition memory, and the performance in a progressive ratio task in adult rats. J. Neuropsychopharmacol. 28, 1760-1769

39 Steinberg, L. and Morris, A.S. (2001) Adolescent development. Annu. Rev. Psychol. 52, $83-110$

40 Mounts, N.S. and Steinberg, L. (1995) An ecological analysis of peer influence on adolescent grade point average and drug use. Dev. Psychol. 31, 915-922

41 Sebastian, C.L. et al. (2010) Social brain development and the affective consequences of ostracism in adolescence. Brain Cogn. $72,134-145$

42 Rigby, K. (2000) Effects of peer victimization in schools and perceived social support on adolescent well-being. J. Adolescence 23, 57-68

43 Einon, D.F. and Morgan, M.J. (1977) A critical period for social isolation in the rat. Dev. Psychobiol. 10, 123-132

44 Ver Hoeve, E.S., et al. (2013) Short-term and long-term effects of repeated social defeat during adolescence or adulthood in female rats. Neuroscience $249,63-73$

45 Birdsong, D. (2006) Age and second language acquisition and processing: a selective overview. Lang. Learn. 56, 9-49

46 Thomas, M.S.C. (2012) Brain plasticity and education. Education. Neurosci. 8, 142-156

47 Kessler, R.C. et al. (2007) Age of onset of mental disorders: a review of recent literature. Curr. Opin. Psychiatry 20, 359-364 
48 Kessler, R.C. et al. (2005) Lifetime prevalence and age-of-onset distributions of DSM-IV disorders in the National Comorbidity Survey Replication. Arch. Gen. Psychiatry 62, 593602

49 Kim-Cohen, J. et al. (2003) Prior juvenile diagnoses in adults with mental disorder: developmental follow-back of a prospective-longitudinal cohort. Arch. Gen. Psychiatry $60,709-717$

50 Sirin, S.R. et al. (2013) The role of acculturative stress on mental health symptoms for immigrant adolescents: a longitudinal investigation. Dev. Psychol. 49, 736-748

51 Pattwell, S.S., et al. (2012) Altered fear learning across development in both mouse and human. PNAS 109, 16318-16323

52 McCormick, C.M., et al. (2013) Age and adolescent social stress effects on fear extinction in female rats. Stress $16,678-688$

53 Buwalda, B., et al. (2013) Adolescent social stress does not necessarily lead to a compromised adaptive capacity during adulthood: a study on the consequences of social stress in rats. Neuroscience $249,258-270$

54 Luciana, M., et al. (2009) Tower of London performance in healthy adolescents: the development of planning skills and associations with self-reported inattention and impulsivity. Dev. Neuropsychol. 34, 461-475

55 Luciana, M., et al. (2005) The development of nonverbal working memory and executive control processes in adolescents. Child Dev. 76, 697-712

56 Germine, L.T., et al. (2011) Where cognitive development and aging meet: face learning ability peaks after age 30 . Cognition $118,201-210$

57 Shing, Y.L., et al. (2010) Episodic memory across the lifespan: the contributions of associative and strategic components. Neurosci. Biobehav. Rev. 34, 1080-1091 
58 Beddeley, A.D. and Hitch, G.J. (1974) Working memory. In Recent advances in learning and motivation (Bower, G.H., ed), pp. 47-89, Academic Press

59 Conklin, H.M., et al. (2007) Working memory performance in typically developing children and adolescents: Behavioral evidence of protracted frontal lobe development. Dev. Neuropsychol. 31, 103-128

60 Jaeggi, S.M., et al. (2011) Short- and long-term benefits of cognitive training. PNAS 108, $10081-10086$

61 Løhaugen, G.C.C., et al. (2011) Computerized working memory training improves function in adolescents born at extremely low birth weight. J. Pediatr. 158, 555-561

62 Rubin, D.C. and Schulkind, M.D. (1997) The distribution of autobiographical memories across the lifespan. Mem. Cognition. 25, 859-866

63 Conway, M.A., et al. (2005) A cross-cultural investigation of autobiographical memory: on the universality and cultural variation of the reminiscence bump. J. Cross-Cult. Psychol. 36, 739-749

64 Janssen, S.M.J., et al. (2008) Reminiscence bump in memory for public events. Eur. J. Cogn. Psychol. 20, 738-764

65 Janssen, S.M.J., et al. (2007) Temporal distribution of favourite books, movies, and records: Differential encoding and re-sampling. Memory 15, 755-767

66 Janssen, S.M.J. and Murre, J.M. (2008) Reminiscence bump in autobiographical memory: Unexplained by novelty, emotionality, valence, or importance of personal events. Quart. J. Exp. Psychol. 60, 1847-1860

67 Murre, J.M., et al. (2013) The rise and fall of immediate and delayed memory for verbal and visuospatial information from late childhood to late adulthood. Acta Psychologica, $142,96-107$ 
35168 Klingberg, T. (2010) Training and plasticity of working memory. TiCS, 14, 317-324

35269 UNICEF (2011) The State of the World's Children 2011: Adolescence - An Age of

$353 \quad$ Opportunity. UNICEF

35470 Melchiorre, A. and Atkins, E. (2011) At what age ate school-children employed, marriend

355 and taken to court? Trends over time. The Right to Education Project

35671 Center on the Developing Child (2010) The foundations of lifelong health are built in

357 early childhood. Harvard University

35872 Allen, D. and Smith, H.I.D. (2009) Early intervention: Good parents, great kids, better

359 citizens. The Centre for Social Justice

36073 World Health Organization (2014) Health for the world's adolescents - A second chance

361 in the second decade. WHO

36274 The Royal Society Policy Centre (2014) Vision for science and mathematics education.

363 The Royal Society 
Figure 1. The effects of cannabis consumption on IQ in adolescence and adulthood. 1,037 participants were followed from birth to age 38. Cannabis dependence was diagnosed in interviews at ages $18,21,26,32$ and 38 . The change in IQ from childhood to adulthood is shown here for participants with 1, 2 or $3+$ diagnoses of cannabis dependence as a function of onset of cannabis dependence. Black bars represent individuals with adolescent-onset cannabis dependence and grey bars individuals with adult-onset cannabis dependence [25].

Figure 2. Models of plasticity in adolescence. Adolescence may be a stand-alone period of

372 heightened plasticity (A) or form a continuous sensitive period with childhood (B). Alternatively, plasticity may decline continuously from childhood through adolescence and into adulthood (C). Adapted from [45, 46].

Figure 3. The interquartile ranges of the age of onset $(\mathrm{AOO})$ of selected psychiatric disorders. The AoO data for Schizophrenia Spectrum Diagnosis was adapted from the Early Psychosis Prevention and Intervention Centre in Melbourne, Australia, as reviewed by Kessler et al. [47]. The AoO for the remaining disorders stems from the National Comorbidity Survey Replication in the United States [48].

Figure 4. The lifespan retrieval curve. The retrieval curve shows a peak of autobiographical memories around adolescence and early adulthood - the reminiscence bump. Adapted from [62]. 